\title{
Excitation of electron cyclotron harmonic waves in the inner Saturn magnetosphere within local plasma injections
}

\author{
X. Tao, ${ }^{1}$ R. M. Thorne, ${ }^{1}$ R. B. Horne, ${ }^{2}$ S. Grimald ${ }^{3,4}$ C. S. Arridge, ${ }^{5,6}$ \\ G. B. Hospodarsky, ${ }^{7}$ D. A. Gurnett, ${ }^{7}$ A. J. Coates, ${ }^{5,6}$ and F. J. Crary ${ }^{8}$ \\ Received 23 April 2010; revised 30 August 2010; accepted 7 September 2010; published 4 December 2010.
}

[1] Strong electron cyclotron harmonic (ECH) waves have been observed by Cassini associated with local plasma injection regions in the Saturnian magnetosphere. Using measured electron distributions from the Cassini plasma spectrometer, we calculated local growth rates and path-integrated wave gain of ECH waves inside an injection event using the HOTRAY code. We showed that electrons with energy near a few $\mathrm{eV}$ have a collision frequency comparable to their bounce frequency; thus, they cannot have an empty loss cone. We then demonstrated that the growth of ECH waves inside the injection event can be driven by electron phase space density gradients associated with the loss cone distribution of injected electrons at energies between a few hundred $\mathrm{eV}$ and a few $\mathrm{keV}$. This conclusion is contrary to previous results that the source of free energy for growth of $\mathrm{ECH}$ waves is provided by electrons near a few eV. Results in this work are helpful for understanding the generation of $\mathrm{ECH}$ waves and their roles in electron dynamics in the Saturnian magnetosphere.

Citation: Tao, X., R. M. Thorne, R. B. Horne, S. Grimald, C. S. Arridge, G. B. Hospodarsky, D. A. Gurnett, A. J. Coates, and F. J. Crary (2010), Excitation of electron cyclotron harmonic waves in the inner Saturn magnetosphere within local plasma injections, J. Geophys. Res., 115, A12204, doi:10.1029/2010JA015598.

\section{Introduction}

[2] Electron cyclotron harmonic $(\mathrm{ECH})$ waves are electrostatic emissions occurring at frequencies between harmonics of the electron gyrofrequency and were previously called $(n+1 / 2)$ $f_{c e}$ waves [Horne et al., 2003]. ECH waves have been found frequently in the magnetospheres of the Earth [Horne et al., 2003; Meredith et al., 2000, 2009], Jupiter [Kurth et al., 1980], and Saturn [Hospodarsky et al., 2008]. In the terrestrial environment, ECH waves could play a potentially important role in the scattering of plasma sheet electrons leading to precipitation into the atmosphere and the excitation of diffuse aurora emissions [Horne et al., 2003; Meredith et al., 2009].

\footnotetext{
${ }^{1}$ Department of Atmospheric and Oceanic Sciences, University of California, Los Angeles, California, USA.

${ }^{2}$ British Antarctic Survey, Cambridge, UK

${ }^{3}$ Centre d'Etude Spatiale des Rayonnements, Université de Toulouse, Toulouse, France.

${ }^{4}$ Centre d'Etude Spatiale des Rayonnements, Université Paul Sabatier, CNRS, Toulouse, France.

${ }^{5}$ Mullard Space Science Laboratory, Department of Space and Climate Physics, University College London, Holmbury St. Mary, UK.

${ }^{6}$ Centre for Planetary Sciences at UCL/Birkbeck, London, UK.

${ }^{7}$ Department of Physics and Astronomy, University of Iowa, Iowa City, Iowa, USA.

${ }^{8}$ Southwest Research Institute, San Antonio, Texas, USA.

Copyright 2010 by the American Geophysical Union.

0148-0227/10/2010JA015598
}

It is possible that $\mathrm{ECH}$ waves might also affect electron dynamics in the Saturnian magnetosphere.

[3] Saturn's magnetosphere is different from the solar wind driven terrestrial magnetosphere because it is rapidly rotating and its convection is driven by the centrifugal force [Hill et al., 1981]. The centrifugal interchange instability reduces the total centrifugal potential energy of the magnetospheric plasma, forcing cold and dense plasma to move radially outward and causing the inward injection of hot and tenuous plasma [e.g., Hill, 1976; Hill et al., 2005]. Evidence for such interchange motions has previously been identified in the Jovian magnetosphere [Bolton et al., 1997; Thorne et al., 1997] and by Cassini at Saturn [e.g., Hill et al., 2005; Burch et al., 2005; Mauk et al., 2005]. ECH waves have been observed both inside and outside injection regions, but with different characteristics. Within injection regions, ECH waves have been shown to be unusually strong in multiple cyclotron harmonic bands, while ECH waves observed outside injection events normally only have a strong first harmonic band and much weaker higher bands [Hospodarsky et al., 2008; Menietti et al., 2008a, 2008b]. In this paper we focus our attention on the excitation of ECH waves within local plasma injection regions.

[4] It is generally acknowledged that the excitation of ECH waves is caused by a loss cone instability [e.g., Ashour Abdalla and Kennel, 1978; Horne et al., 2003]. Menietti et al. [2008b] previously used electron data measured by the electron spectrometer sensor (ELS) of the Cassini plasma spectrometer (CAPS) [Young et al., 2004] to calculate ECH wave growth rates inside an injection event. They con- 


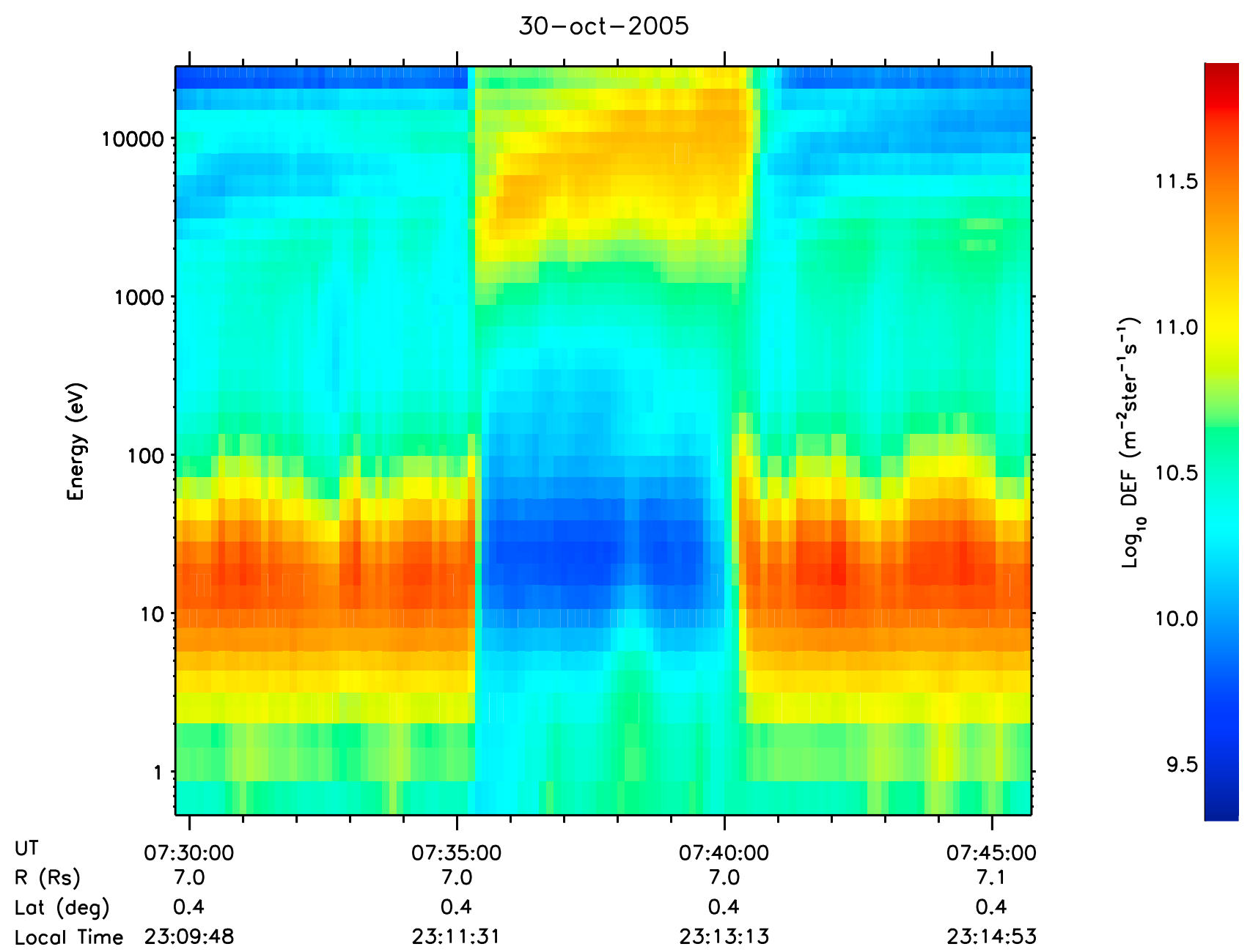

Figure 1. Logarithm of electron differential energy flux $\left(\log _{10} \mathrm{DEF}\right)$ plotted as a function of time and energy around 0737 UT on day 303 of 2005.

clude that a narrow loss cone distribution of electrons with energy near a few eV could provide the phase space density gradient to drive the instability of the Saturnian $\mathrm{ECH}$ waves. However, as we show in section 3, electrons below a few eV have a collision frequency comparable with their bounce frequency and thus should have filled loss cones, contrary to the empty loss cones assumed by Menietti et al. [2008b]. Consequently such electrons near a few eV cannot provide the source of free energy for the growth of ECH waves.

[5] In this work, we revisit the injection event considered by Menietti et al. [2008b]. We use the HOTRAY code [Horne, 1989] and data from CAPS/ELS instrument to calculate local ECH wave growth rates and path integrated wave gain in section 3. First in section 3.1 we compare the collision frequency and the bounce frequency of low energy electrons near a few $\mathrm{eV}$ and demonstrate that these electrons should not have an empty loss cone. A model of the electron phase space density is presented in section 3.2 based on Cassini observations. In section 3.3 we show that loss cones of energetic electrons (few hundred $\mathrm{eV}$ to a few $\mathrm{keV}$ ) provide a viable source to drive instability of $\mathrm{ECH}$ waves inside the injection event. Finally, we summarize our results and discuss future work in section 4 .

\section{Electron Distributions and ECH Waves From Observation}

[6] The local plasma injection event around 0737 UT on day 303 of year 2005 has been studied by several authors [Menietti et al., 2008b; Rymer et al., 2008]. In this work, we use electron measurements from CAPS/ELS [Young et al., 2004], which measures electrons in the energy range of $0.6 \mathrm{eV}$ to $28 \mathrm{keV}$, with an instantaneous field of view of $5.2^{\circ} \times 160^{\circ}$. Figure 1 shows a depletion of cold electrons and an enhancement of $\mathrm{keV}$ electrons observed by CAPS/ ELS when the spacecraft encounters the local plasma injection event between about 0735 and 0740 UT at $L=7$. The phase space density of electrons during the injection event at 0737 UT, shown in Figure 2, exhibits a pancake distribution (peaked near $90^{\circ}$ pitch angle) for energetic electrons $(E>$ a few $\mathrm{keV})$. The phase space density of lower energy electrons $(E<100 \mathrm{eV})$ is more field-aligned during the injection event at 0737 UT. These results are consistent with those of Rymer et al. [2008]. 


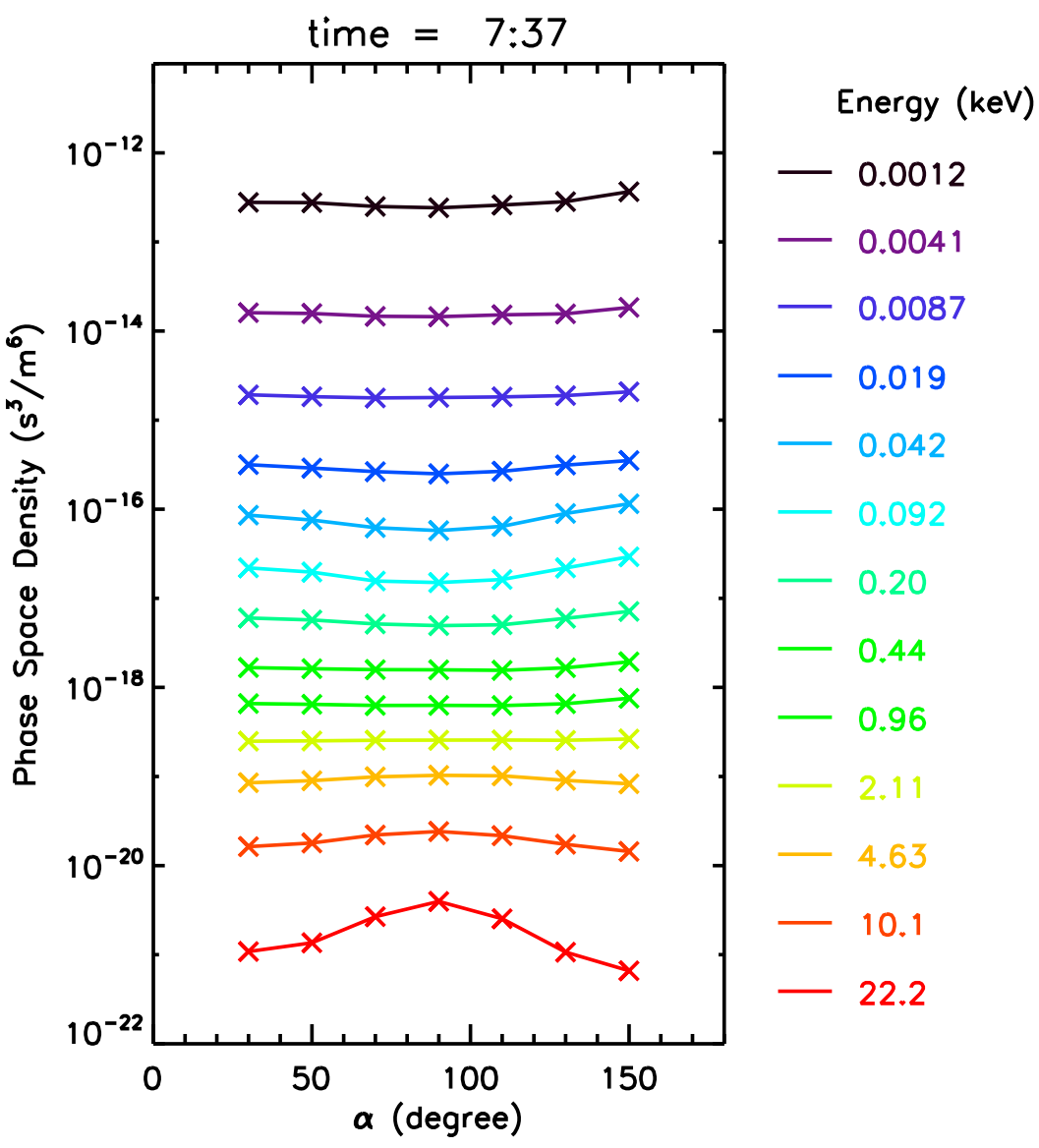

Figure 2. Phase space densities of electrons from CAPS/ELS measurements at selected energies (different colors) at 0737 UT inside the injection event. Crosses represent actual data points.

[7] During the local plasma injection event, strong ECH waves with several harmonic bands are observed, as shown in Figure 3. Figure 4 shows the electric field power spectral density obtained from the medium frequency receiver (MFR) of the Cassini radio and plasma wave science (RPWS) instrument [Gurnett et al., 2004] as a function of frequency at 0737 UT on day 303 of 2005 . The electron cyclotron frequency is determined using data provided by Cassini magnetometer team (MAG) [Dougherty et al., 2004]. Particularly notable is the intense power spectral densities of the second to the fourth band ECH waves, which are comparable to that of the first band.

\section{ECH Wave Growth Inside the Local Plasma Injection Event}

\subsection{Loss Cone Depth and Width}

[8] The loss cone width and depth have been shown to be crucial for the evaluation of ECH wave growth rates and wave gain [Ashour-Abdalla and Kennel, 1978; Horne et al., 2003]. It is important to model the loss cone width and depth as accurate as possible; however, these quantities cannot be obtained from available data. The loss cone is less than $\sim 3^{\circ}$ as determined by Menietti et al. [2008b], thus it is too narrow to be observed by CAPS/ELS. The method adopted in this study to determine whether a loss cone feature should be present is to compare the electron's col- lision frequency $\nu_{c}$ with its bounce frequency $\nu_{B}$. Electrons with $\nu_{c} \geq \nu_{B}$ should have filled loss cones due to collisional scattering, while electrons with $\nu_{c} \ll \nu_{B}$ should exhibit an atmosphere loss cone distribution.

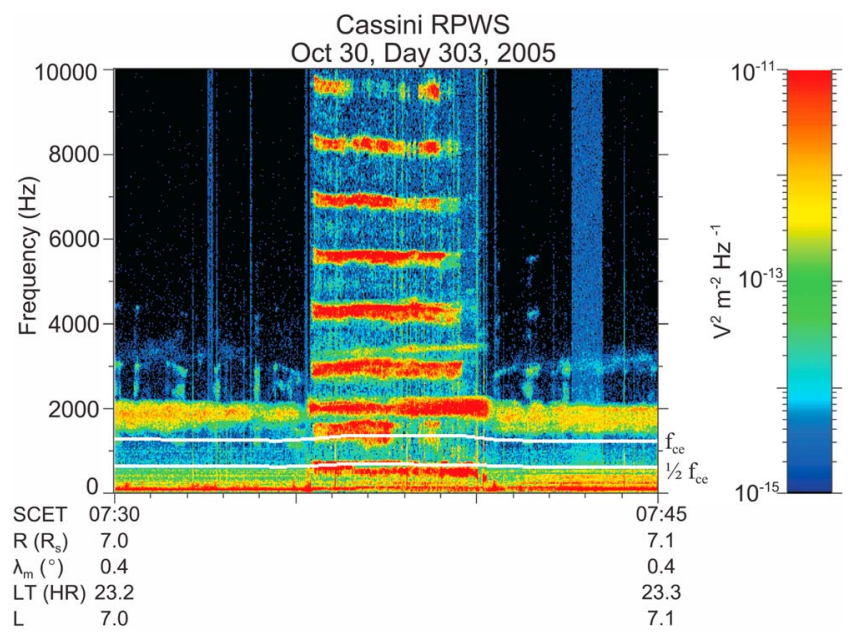

Figure 3. Adapted from Figure 5 of Hospodarsky et al. [2008] showing time-frequency spectrograms of ECH emissions during the injection event around 0737 UT on day 303 of 2005 . White lines indicate the electron gyrofrequency $f_{\text {ce }}$ and $1 / 2 f_{\text {ce }}$, as labeled. 


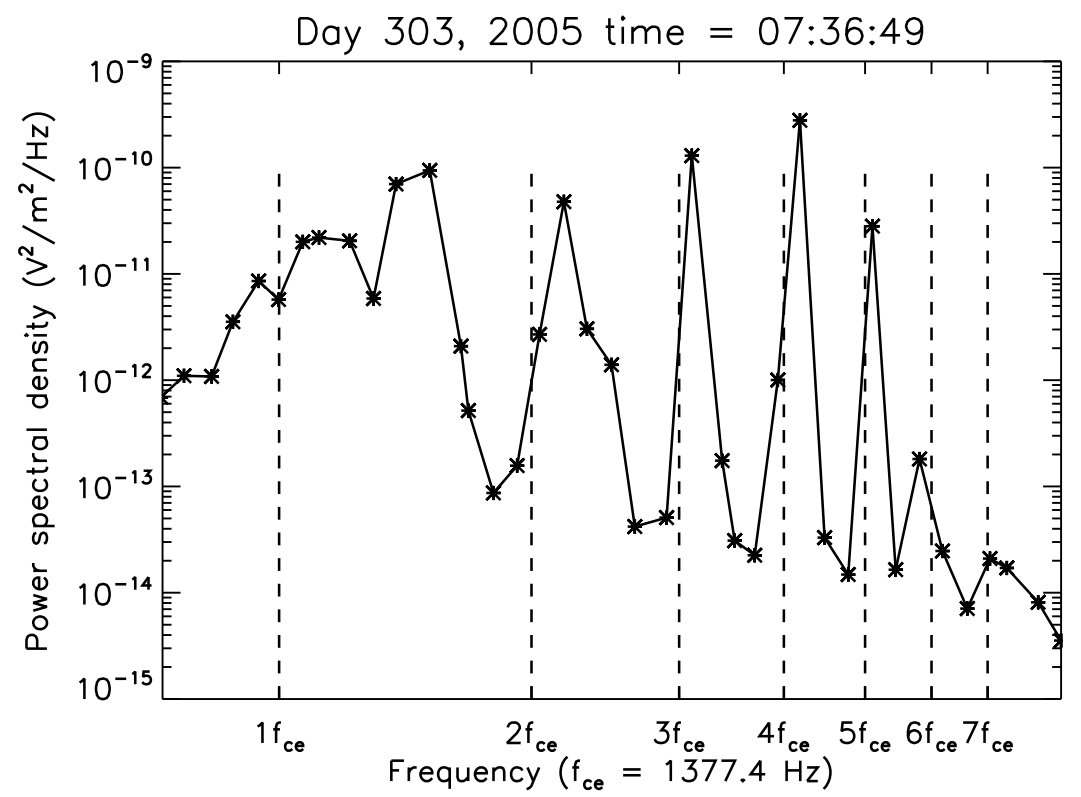

Figure 4. Electric field power spectral density (solid lines) plotted as a function of frequency inside the injection event at 0737 UT on day 303 of 2005. Stars indicate actual data points, and the vertical dotted lines represent harmonics of electron gyrofrequency.

[9] The electron collision frequency due to small angle collisions is (in Gaussian units)

$$
\nu_{\mathrm{c}} \approx \frac{8 \pi n_{\mathrm{e}} q_{\mathrm{e}}^{4} \ln \Lambda}{m_{\mathrm{e}}^{2} \nu_{\mathrm{e}}^{3}},
$$

where $v_{\mathrm{e}}$ is electron's velocity, $n_{\mathrm{e}}$ is the number density, and the electron plasma parameter $\Lambda$ is the number of electrons in a Debye cube [Nicholson, 1992, equation (1.49)]. Numerically, $\Lambda$ is related to the electron temperature $T_{e}$ and number density $n_{e}$ by $\Lambda=4 \times 10^{8} T_{e}^{3 / 2}(\mathrm{eV}) / n_{e}^{1 / 2}\left(\mathrm{~cm}^{-3}\right)$. Because $\ln \Lambda$ is a slowly varying function of $\Lambda$, we use $\ln$ $\Lambda \approx 19$ for $T_{e}=1 \mathrm{eV}$ and $n_{e}=4 \mathrm{~cm}^{-3}$. From equation (1), we can approximate the electron collision frequency as a function of $n_{e}$ and kinetic energy $E$ as $\nu_{c} \approx 4.8 \times 10^{-4} n_{e}$ $\left(\mathrm{cm}^{-3}\right) E^{-3 / 2}(\mathrm{eV})$. Assuming a dipole field, the bounce frequency of an electron is a slowly varying function of its equatorial pitch angle, and may be approximated by $\nu_{B} \approx \sqrt{2 m_{e} E} /\left(4 m_{e} a L\right)$, where $a$ is the Saturn radius and $L$ is the Roederer $L$ [Schulz and Lanzerotti, 1974]. Numerically, $\nu_{B} \approx 2.3 \times 10^{-3} \sqrt{E(\mathrm{eV})} / \mathrm{L}$. Using $n_{e}=4.96 \mathrm{~cm}^{3}$ as determined from the upper hybrid resonance line from wave observation [Hospodarsky et al., 2008] and setting $\nu_{c}=\nu_{B}$, we obtain a critical energy $E^{*}$, below which collisions dominate; $E^{*} \approx 3 \mathrm{eV}$ at $L=7$. Consequently, electrons of a few $\mathrm{eV}$ energy should have filled loss cones due to collisions. Another factor we considered is that the pitch angle distributions of cold electrons $(E<100 \mathrm{eV})$ tend to be field-aligned and have a peak at $0^{\circ}$ and $180^{\circ}$ [Rymer et al., 2008]. These electrons $(E<100 \mathrm{eV})$ probably originate from the atmosphere and would be expected to have essentially filled loss cones. Based on the above reasoning, we only assign loss cone distributions to electrons with $E>100 \mathrm{eV}$ in the following calculation.

[10] We note that our loss cone features are different from that of Menietti et al. [2008b], where all electron popula- tions have been given an empty loss cone. As shown below, this difference in the electron distribution below $100 \mathrm{eV}$ gives us a fundamentally different conclusion from that of Menietti et al. [2008b] on which electron population drives ECH wave growth inside the injection event.

\subsection{Modeling Observed Electron Distribution Inside the Injection Event}

[11] The HOTRAY code [Horne, 1989] is used to calculate wave growth rates and path integrated wave gain. The HOTRAY code requires electron distributions to be modeled by a summation of subtracted bi-Maxwellians [Ashour-Abdalla and Kennel, 1978] of the form

$$
\begin{aligned}
f\left(v_{\perp}, v_{\|}\right)= & \sum_{i} \frac{n_{i}}{\pi^{3 / 2} \alpha_{\perp i}^{2} \alpha_{\| i}} \exp \left(-\frac{\left(v_{\|}-v_{d i}\right)^{2}}{\alpha_{\| i}^{2}}\right) \\
& {\left[\Delta_{i} \exp \left(-\frac{v_{\perp}^{2}}{\alpha_{\perp i}^{2}}\right)+\frac{\left(1-\Delta_{i}\right)}{\left(1-\beta_{i}\right)}\right.} \\
& \left.\left(\exp \left(-\frac{v_{\perp}^{2}}{\alpha_{\perp i}^{2}}\right)-\exp \left(-\frac{v_{\perp}^{2}}{\beta_{i} \alpha_{\perp i}^{2}}\right)\right)\right] .
\end{aligned}
$$

Here $\Delta_{i}$ specifies the depth of the loss cone $\left(\Delta_{i}=0\right.$ means an empty loss cone), $\beta_{i}$ is the width of the loss cone, $\alpha_{\perp i}$ and $\alpha_{\| i}$ are the thermal velocity perpendicular and parallel to the local magnetic field, respectively, $v_{d i}$ is the drift velocity along the magnetic field line, and $n_{i}$ is the number density.

[12] We fit data observed by CAPS/ELS at 0737 UT on day 303, 2005 using a nonlinear least squares fitting with IDL routine MPFIT [Markwardt, 2009] using eight components, and plot the resulting fitting function together with data in Figure 5. The total electron density from CAPS/ELS measurement is smaller than that from the number density determined from the upper hybrid resonance line by about a factor of two, probably because of a negative spacecraft potential and a limited energy range of CAPS/ELS. Con- 

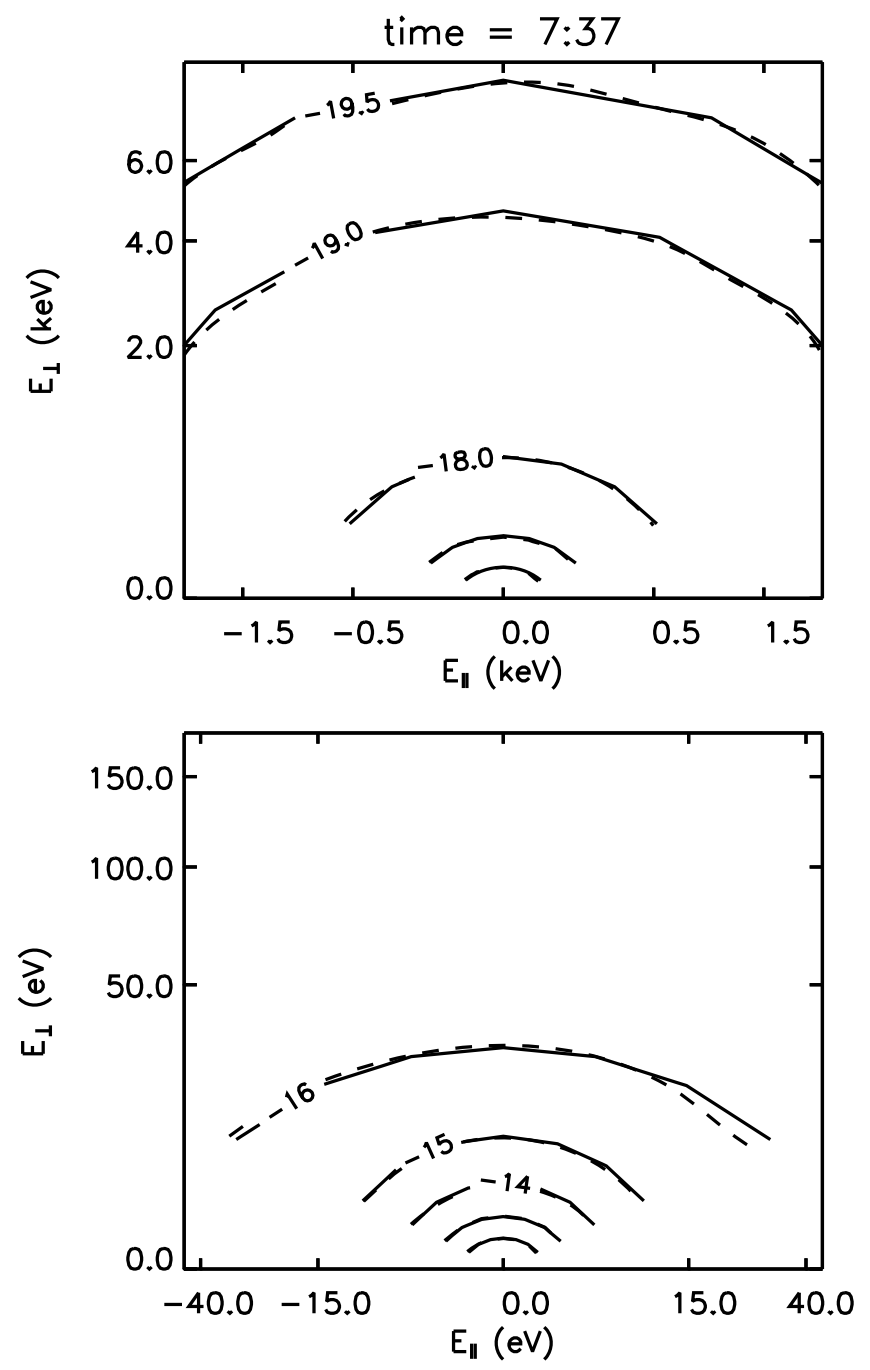

Figure 5. Contours of the logarithm of electron phase space densities $\left(\log _{10} f\right)$ inside the injection event at 0737 UT as a function of perpendicular energy $\left(E_{\perp}\right)$ and parallel energy $\left(E_{\|}\right)$from CAPS/ELS data (dotted lines) and our modeled distribution (solid lines): (top) higher energy electrons and (bottom) lower energy electrons.

sequently, we modify the number density of the coldest component so that the total number density is equal to that from plasma wave measurements $\left(n_{\text {tot }} \approx 4.96 \mathrm{~cm}^{-3}\right)$ [Gurnett et al., 2005; Persoon et al., 2005]. The resulting fitting parameters are shown in Table 1.

\subsection{Wave Growth Rates and Path Integrated Wave Gain}

[13] The wave growth rates of the first seven cyclotron harmonic bands are calculated using the dispersion solver of HOTRAY. The calculations are performed at $\mathrm{L}=7.8$, where the magnetic field given by our dipole field model is equal to the magnetic field from MAG measurement, to allow accurate calculation of the electron resonant energies. The results, shown in Figure 6, indicate that ECH waves in all seven harmonic bands can be excited. We note that for the first band, there are two peaks in growth rates at $1.36 \Omega_{c e}$ and $1.79 \Omega_{c e}$. The peak at $1.79 \Omega_{c e}$ has a wave vector $k=0.02 \mathrm{~m}^{-1}$. From these parameters, we calculate the lowest parallel resonant energy to be about $10 \mathrm{keV}$ using the resonant condition $\omega-k_{\|} \nu_{\|}=n \Omega_{c e}$, where $\omega$ is the angular wave frequency, $\Omega_{c e}$ is the electron angular gyrofrequency, $k_{\|}$ is the wave vector component that is parallel to the ambient magnetic field, and $\nu_{\|}$is the parallel electron velocity. The second peak at $1.36 \Omega_{c e}$ has a $k=0.05 \mathrm{~m}^{-1}$, which corresponds to the lowest parallel resonant energy of about $300 \mathrm{eV}$. Thus, both electrons near a few hundred $\mathrm{eV}$ and a few $\mathrm{keV}$ contribute to growth of ECH waves inside the injection event.

[14] During propagation, the wave normal angle and the ambient magnetic field changes, which results in changes of resonant energies. We consequently use HOTRAY to calculate the path integrated wave gain to take into account the propagation effects. The path-integrated wave growth rate in HOTRAY is calculated as $\Gamma \approx \Sigma k_{i} \cdot \Delta R$, where $\Delta \boldsymbol{R}$ is the distance along the raypath and $\boldsymbol{k}_{i}$ is the spatial growth rate, i.e., the imaginary part of wave vector $\boldsymbol{k} \equiv \boldsymbol{k}_{r}+\mathrm{i} \boldsymbol{k}_{i}$ as determined from the dispersion relation. The net wave gain is then given by $G=20 \log _{10}\left(\mathrm{e}^{\Gamma}\right) \approx 8.69 \Gamma \mathrm{dB}$.

[15] ECH rays from each of the seven bands with maximum wave gain at the given frequency and wave normal angle are shown in Figure 7 as a function of group time, calculated using HOTRAY assuming the electron distributions to be constant as shown in Table 1. The group time is the independent variable used in the HOTRAY code [Horne, 1989], referring to the time for energy propagation, and it is appropriate for comparison with observation [Horne et al., 2003]. Note that we limit the longitudinal width of the propagation path to be less than 2.8 degrees, which is the longitudinal width of the injection event estimated by $\Omega_{\mathrm{s}} \Delta_{t}$, following Hill et al. [2005]. Here $\Omega_{\mathrm{s}} \approx 2 \pi /$ $10.8 \mathrm{~h}$ is the angular rotation frequency of Saturn, and $\Delta t \approx$ $5 \mathrm{~min}$ is the duration of the local injection event observed by Cassini as shown in Figure 1. From Figure 7, we see that ECH waves can obtain substantial wave gain $(\sim 60-80 \mathrm{~dB})$ before they reach the boundary of the injection region. This demonstrates that injected hot electrons with energy larger than a few hundred $\mathrm{eV}$ can drive the excitation of $\mathrm{ECH}$ waves inside this injection event. The raypath plot indicates that ECH waves are strongly confined to the equatorial region and that they reflect about the equatorial plane similar to ECH waves at Earth [Horne et al., 2003]. These rays can also move radially inward, consequently the wave frequency becomes closer to harmonics of the local electron cyclotron frequency. For example, the ray with $\omega / \Omega_{c e}(L=7.8)=1.5$ moves inward by as far as $0.8 \mathrm{R}_{\mathrm{s}}$, thus its frequency normalized to local $\Omega_{c e}$ at $L=7$ becomes $\omega / \Omega_{c e}(L=7) \approx 1.08$.

Table 1. Fitting Parameters of Measured Electron Distribution Inside an Injection Event at 0737 UT Using Eight Subtracted Bi-Maxwellian Functions

\begin{tabular}{cccccc}
\hline Component & $\Delta$ & $T_{\perp}(\mathrm{keV})$ & $T_{\|}(\mathrm{keV})$ & $\beta$ & $n\left(\mathrm{~m}^{-3}\right)$ \\
\hline 1 & 1 & $2.70 \times 10^{-4}$ & $3.42 \times 10^{-4}$ & 0 & $4.56 \times 10^{6}$ \\
2 & 1 & $1.04 \times 10^{-3}$ & $1.13 \times 10^{-3}$ & 0 & $1.18 \times 10^{5}$ \\
3 & 1 & $3.28 \times 10^{-3}$ & $3.40 \times 10^{-3}$ & 0 & $1.39 \times 10^{5}$ \\
4 & 1 & $1.38 \times 10^{-2}$ & $2.23 \times 10^{-2}$ & 0 & $4.36 \times 10^{4}$ \\
5 & 1 & $5.78 \times 10^{-2}$ & $8.04 \times 10^{-2}$ & 0 & $3.35 \times 10^{4}$ \\
6 & 0 & $3.00 \times 10^{-1}$ & $3.52 \times 10^{-1}$ & 0.0005 & $2.96 \times 10^{4}$ \\
7 & 0 & 2.40 & 2.18 & 0.001 & $7.48 \times 10^{4}$ \\
8 & 0 & 9.80 & 5.32 & 0.001 & $3.03 \times 10^{4}$ \\
\hline
\end{tabular}



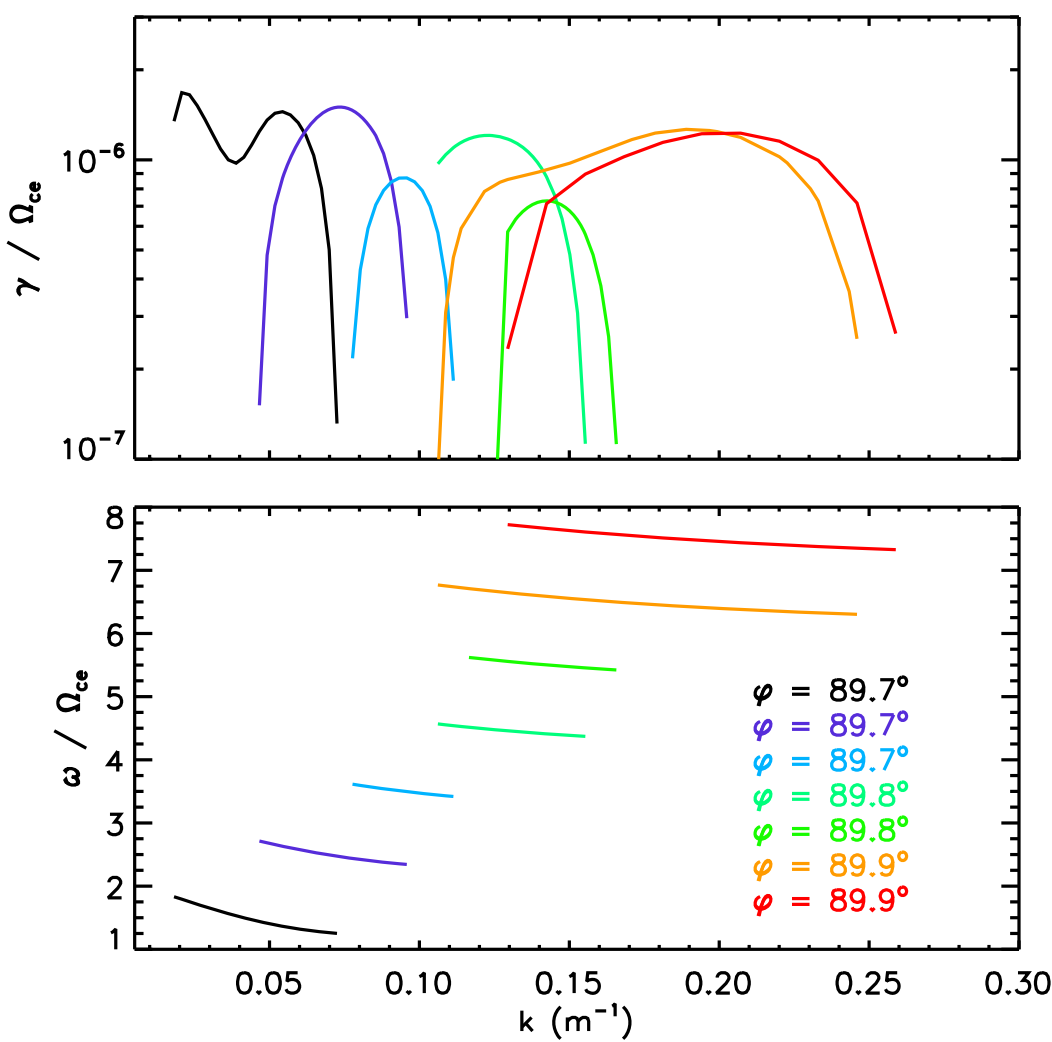

Figure 6. Imaginary part $(\gamma)$ and real part $(\omega)$ wave frequency, normalized by electron gyrofrequency $\left(\Omega_{\mathrm{ce}}\right)$, as a function of wave vector $k$ for seven band ECH waves with different wave normal angles $\varphi$ (indicated by different colors) inside the injection event at time 0737 UT. 

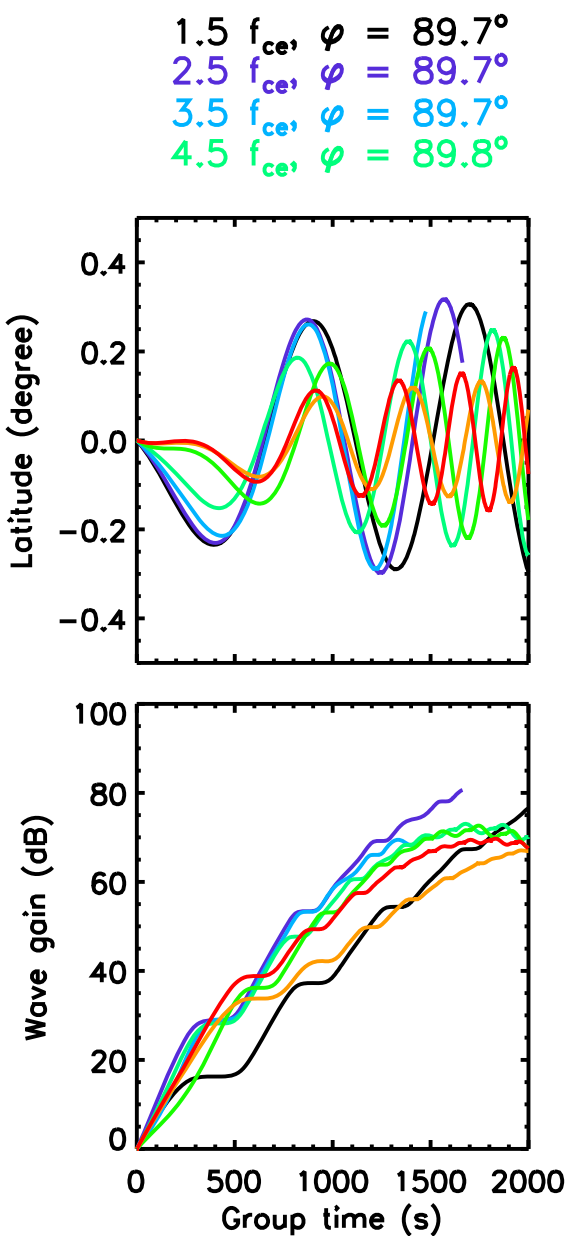
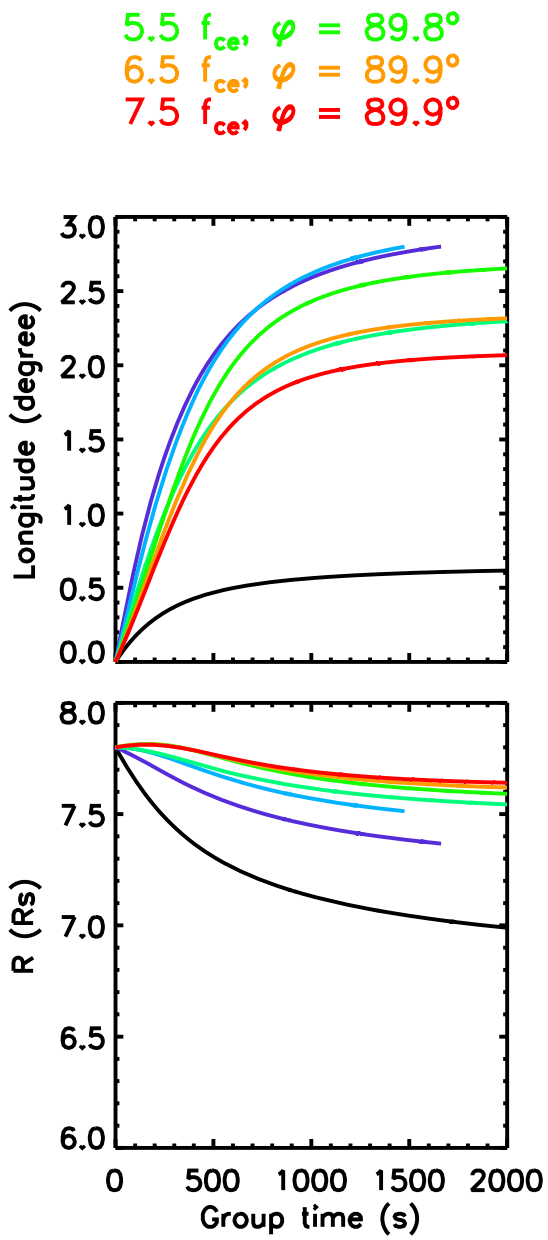

Figure 7. (top left) Latitude, (top right) longitude, (bottom right) radial distance, and (bottom left) wave gain of seven rays from seven ECH bands with different wave normal angles $\varphi$ (represented by different colors), plotted as a function of group time for the ECH waves at 0737 UT.

This feature of ECH rays might be responsible for the observation that some ECH waves have frequencies very close to harmonics of the local electron cyclotron frequency (e.g., see Figures 3 and 4).

\section{Summary}

[16] In this work, electron distributions measured by CAPS/ELS on Cassini have been used to calculate wave growth rates and path-integrated wave gain of ECH waves inside an injection event around 0737 UT on day 303 of 2005. Using the HOTRAY code, wave growth rates and path integrated wave gain were calculated and it was demonstrated that ECH waves from all seven bands could obtain substantial wave gain $(\sim 60-80 \mathrm{~dB})$ inside the injection region. Electrons with energy near a few hundred $\mathrm{eV}$ and few $\mathrm{keV}$ contribute to $\mathrm{ECH}$ wave growth inside the injection event. This result is consistent with simultaneous observation of strong ECH emissions and a pronounced enhancement of hot electron fluxes during inward moving plasma injection events. Thus, for ECH waves inside injection events, free energy for wave growth comes from energetic electrons with energy larger than a few hundred $\mathrm{eV}$, instead of cold electrons with a few eV energy, as earlier suggested by Menietti et al. [2008a] and Menietti et al. [2008b]. The precise ECH wave gain, however, is very sensitive to the electron distribution, such as the temperature of the coldest component [Horne et al., 1981] and loss cone depth and width [Horne et al., 2003]. We note that the use of a simple empty loss cone model might not be realistic and there are also some uncertainties in modeling cold electron components in our current analysis. We leave determination of $\mathrm{ECH}$ wave gain using better loss cone information and cold electron distributions to future work.

[17] Acknowledgments. We would like to thank the Cassini team for making the data available through the PPI node of the Planetary Data System and Binbin Ni for helpful discussions. Sandrine Grimald acknowledges financial support from CNES. CSA was supported in this work by an STFC postdoctoral fellowship under grant ST/G007462/1. AJC and SG were supported by the STFC rolling grant to MSSL/UCL. Cassini data processing was supported in the UK by STFC. The research at The University of Iowa is supported by the National Aeronautics and Space Administration through contract 1356500 with the Jet Propulsion Laboratory. The work at University of California-Los Angeles was supported by NASA grant NNX08AP80G.

[18] Masaki Fujimoto thanks the reviewers for their assistance in evaluating this paper.

\section{References}

Ashour-Abdalla, M., and C. F. Kennel (1978), Nonconvective and convective electron cyclotron harmonic instabilities, J. Geophys. Res., 83(A4), 1531-1543, doi:10.1029/JA083iA04p01531. 
Bolton, S. J., R. M. Thorne, D. A. Gurnett, W. S. Kurth, and D. J. Williams (1997), Enhanced whistler-mode emissions: Signatures of interchange motion in the Io torus, Geophys. Res. Lett., 24(17), 2123-2126, doi:10.1029/97GL02020.

Burch, J. L., J. Goldstein, T. W. Hill, D. T. Young, F. J. Crary, A. J. Coates, N. André, W. S. Kurth, and E. C. Sittler Jr. (2005), Properties of local plasma injections in Saturn's magnetosphere, Geophys. Res. Lett., 32, L14S02, doi:10.1029/2005GL022611.

Dougherty, M. K., et al. (2004), The Cassini magnetic field investigation, Space Sci. Rev., 114, 331-383, doi:10.1007/s11214-004-1432-2.

Gurnett, D. A., et al. (2004), The Cassini radio and plasma wave investigation, Space Sci. Rev., 114, 395-463, doi:10.1007/s11214-004-1434-0.

Gurnett, D. A., et al. (2005), Radio and plasma wave observations at Saturn from Cassini's approach and first orbit, Science, 307, 1255-1259, doi:10.1126/science. 1105356 .

Hill, T. W. (1976), Interchange stability of a rapidly rotating magnetosphere, Planet. Space Sci., 24, 1151-1154, doi:10.1016/0032-0633(76)90152-5.

Hill, T. W., A. J. Dessler, and L. J. Maher (1981), Corotating magnetospheric convection, J. Geophys. Res., 86(A11), 9020-9028, doi:10.1029/ JA086iA11p09020.

Hill, T. W., A. M. Rymer, J. L. Burch, F. J. Crary, D. T. Young, M. F. Thomsen, D. Delapp, N. André, A. J. Coates, and G. R. Lewis (2005), Evidence for rotationally driven plasma transport in Saturn's magnetosphere, Geophys. Res. Lett., 32, L14S10, doi:10.1029/2005GL022620.

Horne, R. B. (1989), Path-integrated growth of electrostatic waves: The generation of terrestrial myriametric radiation, J. Geophys. Res., 94(A7), 8895-8909, doi:10.1029/JA094iA07p08895.

Horne, R. B., P. J. Christiansen, M. P. Gough, K. Rönnmark, J. F. E. Johnson, J. Sojka, and G. L. Wrenn (1981), Amplitude variations of electron cyclotron harmonic waves, Nature, 294, 338-340, doi:10.1038/294338a0.

Horne, R. B., R. M. Thorne, N. P. Meredith, and R. R. Anderson (2003), Diffuse auroral electron scattering by electron cyclotron harmonic and whistler mode waves during an isolated substorm, J. Geophys. Res., 108(A7), 1290, doi:10.1029/2002JA009736.

Hospodarsky, G. B., T. F. Averkamp, W. S. Kurth, D. A. Gurnett, J. D. Menietti, O. Santolik, and M. K. Dougherty (2008), Observations of chorus at Saturn using the Cassini Radio and Plasma Wave Science instrument, J. Geophys. Res., 113, A12206, doi:10.1029/2008JA013237.

Kurth, W. S., D. D. Barbosa, D. A. Gurnett, and F. L. Scarf (1980), Electrostatic waves in the Jovian magnetosphere, Geophys. Res. Lett., 7(1), 57-60, doi:10.1029/GL007i001p00057.

Markwardt, C. B. (2009), Nonlinear least-squares fitting in IDL with MPFIT, in Astronomical Data Analysis Software and Systems XVIII, edited by D. Bohlender, P. Dowler, and D. Durand, Astron. Soc. Pac. Conf. Ser., 411, 251-254.

Mauk, B. H., et al. (2005), Energetic particle injections in Saturn's magnetosphere, Geophys. Res. Lett., 32, L14S05, doi:10.1029 2005GL022485.

Menietti, J. D., O. Santolik, A. M. Rymer, G. B. Hospodarsky, D. A. Gurnett, and A. J. Coates (2008a), Analysis of plasma waves observed in the inner
Saturn magnetosphere, Ann. Geophys., 26(9), 2631-2644, doi:10.5194/ angeo-26-2631-2008.

Menietti, J. D., O. Santolik, A. M. Rymer, G. B. Hospodarsky, A. M. Persoon, D. A. Gurnett, A. J. Coates, and D. T. Young (2008b), Analysis of plasma waves observed within local plasma injections seen in Saturn's magnetosphere, J. Geophys. Res., 113, A05213, doi:10.1029/2007JA012856.

Meredith, N. P., R. B. Horne, A. D. Johnstone, and R. R. Anderson (2000), The temporal evolution of electron distributions and associated wave activity following substorm injections in the inner magnetosphere, J. Geophys. Res., 105(A6), 12,907-12,917, doi:10.1029/2000JA900010.

Meredith, N. P., R. B. Horne, R. M. Thorne, and R. R. Anderson (2009), Survey of upper band chorus and $\mathrm{ECH}$ waves: Implications for the diffuse aurora, J. Geophys. Res., 114, A07218, doi:10.1029/2009JA014230.

Nicholson, D. R. (1992), Introduction to Plasma Theory, Krieger, Malabar, Fla.

Persoon, A. M., D. A. Gurnett, W. S. Kurth, G. B. Hospodarsky, J. B. Groene, P. Canu, and M. K. Dougherty (2005), Equatorial electron density measurements in Saturn's inner magnetosphere, Geophys. Res. Lett., 32, L23105, doi:10.1029/2005GL024294.

Rymer, A. M., B. H. Mauk, T. W. Hill, C. Paranicas, D. G. Mitchell, A. J. Coates, and D. T. Young (2008), Electron circulation in Saturn's magnetosphere, J. Geophys. Res., 113, A01201, doi:10.1029/2007JA012589.

Schulz, M., and L. J. Lanzerotti (1974), Particle Diffusion in the Radiation Belts, Phys. Chem. Space, vol. 7, Springer, New York.

Thorne, R. M., T. P. Armstrong, S. Stone, D. J. Williams, R. W. McEntire, S. J. Bolton, D. A. Gurnett, and M. G. Kivelson (1997), Galileo evidence for rapid interchange transport in the Io torus, Geophys. Res. Lett., 24(17), 2131-2134, doi:10.1029/97GL01788

Young, D. T., et al. (2004), Cassini plasma spectrometer investigation, Space Sci. Rev., 114(1-4), 1-112, doi:10.1007/s11214-004-1406-4.

C. S. Arridge and A. J. Coates, Mullard Space Science Laboratory, Department of Space and Climate Physics, University College London, Holmbury St. Mary RH5 6NT, UK.

F. J. Crary, Southwest Research Institute, PO Box 28510, San Antonio, TX 78288, USA.

S. Grimald, Centre d'Etude Spatiale des Rayonnements, Université Paul Sabatier, CNRS, F-31028 Toulouse, France.

D. A. Gurnett and G. B. Hospodarsky, Department of Physics and Astronomy, University of Iowa, Van Allen Hall, Iowa City, IA 52242-1479, USA.

R. B. Horne, British Antarctic Survey, Madingley Road, Cambridge CB3 OET, UK

X. Tao and R. M. Thorne, Department of Atmospheric and Oceanic Sciences, University of California, 405 Hilgard Ave., 7115 Math Sciences Bldg., Los Angeles, CA 90095, USA. (xtao@atmos.ucla.edu; rmt@atmos.ucla.edu) 